\title{
25.1: Luminance Probes for Contrast Measurements in Medical Displays
}

\author{
Aldo Badano, Scott Pappada, Edward F. Kelley \\ Michael J. Flynn, Sandrine Martin and Jerzy Kanicki
}

\begin{abstract}
We report on a comparative study that examines four conic luminance probes in their ability to measure small-spot display contrast. We performed linear scans of a slit using a computer-controlled stage. We found that, although the probes were assembled according to the same design, small differences in their performance due to minor variations in materials and surfaces can be measured.
\end{abstract}

\section{INTRODUCTION}

Many radiology departments and laboratories are using routinely high performance display devices for primary interpretation of diagnostic images, as well as for review and consultation by referring physicians. This phenomenon is happening in many areas of the radiology practice including digital mammography, general radiography, computedtomography and radionuclide imaging. The number of medical imaging workstations in the United States is estimated to be on the order of 150,000 . In a recent internet poll of 299 radiological departments [1], it was found that $54 \%$ of readings are performed using light-boxes, $33 \%$ are performed using cathode-ray tube (CRT) monitors, and 12\% are carried out using flat panel liquid-crystal (LCD) monitors. The number of readings performed with electronic displays will continue to increase in the next years due to the deployment of digital technologies for the acquisition, storage and transmission of diagnostic images across modalities, and the use of computerized aids for the detection and diagnosis of malignancies. As a result, there exists a need for

AB (agb@cdrh.fda.gov) is with the Medical Imaging and Computer Applications Branch, Center for Devices and Radiological Health, U.S. Food and Drug Administration. SP is with the Department of Biomedical Engineering, Marquette University. EFK is with the Electricity Division, Electronics and Electrical Engineering Laboratory, Technology Administration, U.S. Department of Commerce. MJF is with the Department of Radiology, Henry Ford Health System. JK and SM are with the Department of Electrical Engineering and Computer Science, University of Michigan. This is a contribution of the National Institute of Standards and Technology and of the Food and Drug Administration, and is not subject to copyright. accurate and precise methods to quantify display image quality as it pertains to the way trained human observers perform the visual tasks involved in the medical diagnostic process.

The assessment of the display image quality has been the focus of a recent effort by the American Association of Physicists in Medicine [2]. The display contrast response can be measured with different scenes that include bright surroundings and large variation between the target luminance and the background luminance. The measurement of contrast ratios under those conditions provide, for instance, detailed characterization of veiling glare processes in cathode-ray tubes [3]-[5], and electronic crosstalk patterns in high-resolution activematrix liquid-crystal displays [6], [7].

In previous work, we showed that a collimated probe is required to accurately measure the smallspot contrast ratio of high performance display devices [8]. In the proposed design, the contamination of the luminance measurements by bright surroundings is minimized through careful selection of the reflective properties of internal and external surfaces and the precise localization of baffles. This particular design approach was used by each of the participating laboratories to assemble their own probes. The purpose of this work is to characterize and compare the performance of these probes. Our goal is to verify that the design can be used to fabricate probes with similar performance, while investigating how minor changes in the materials and fabrication methods affect the ability of the probes to measure small-spot contrast ratios.

\section{Methods}

In this work, we compare four probes constructed after the design described in Ref. 8. A simplified diagram of the main aspects of that design is shown in Fig. 2. Probe $\mathrm{A}$ is the device assembled in 


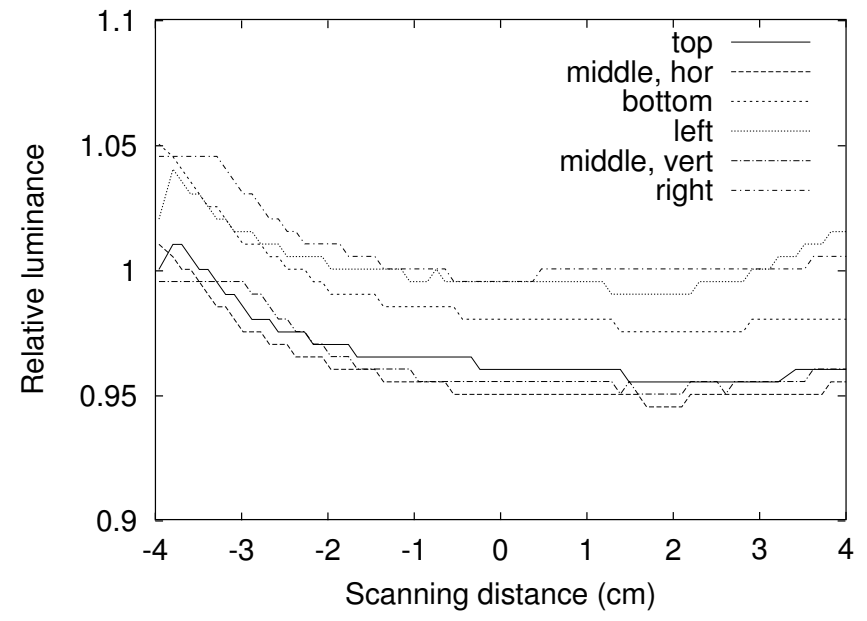

Fig. 1. Luminance uniformity across the center of the light source. The maximum luminance variation for a given scan is below 0.05 .

conjunction with the design work [8]. Probe B was constructed using black plastic and a similar approach but with an additional $5 \mathrm{~cm}$ hood between the conic probe and the detector. Probe $\mathrm{C}$ is a similar probe constructed in a different laboratory, according to the instructions given in Ref. 8. Finally, probe $\mathrm{D}$ was constructed according to a different design based on black plastic with internal frustums acting as baffles [9]. The comparison between the probes was carried out using the same Si high-gain photodiode detector, photopic filter, and reader, so that any difference observed in the results can be attributed to variations in the design or assembly techniques or to differences in material or surface optical properties for each of the four probes (A-D).

The light source used in the experiments was made out of a Styrofoam [10] box $(38.1 \mathrm{~cm} \times$ $38.1 \mathrm{~cm} \times 38.1 \mathrm{~cm}$ ) with a $10 \mathrm{~cm} \times 10 \mathrm{~cm}$ front aperture. The design of this uniform light source is based on Ref. 11. The uniform light source has four halogen lamps inside, which are supplied with a constant $12 \mathrm{~V}$ source. The uniformity of the light source was verified by taking measurements of luminance at the center, upper, and lower section of the aperture using the same scanning technique used throughout this work. As shown in Fig. 1, the maximum variation in relative luminance for a given scan was below 0.05 . The external surfaces of the uniform light source were painted black to minimize any accessory light leakage. LabView [10] programming was used to acquire luminance readings while controlling the motion of the probe

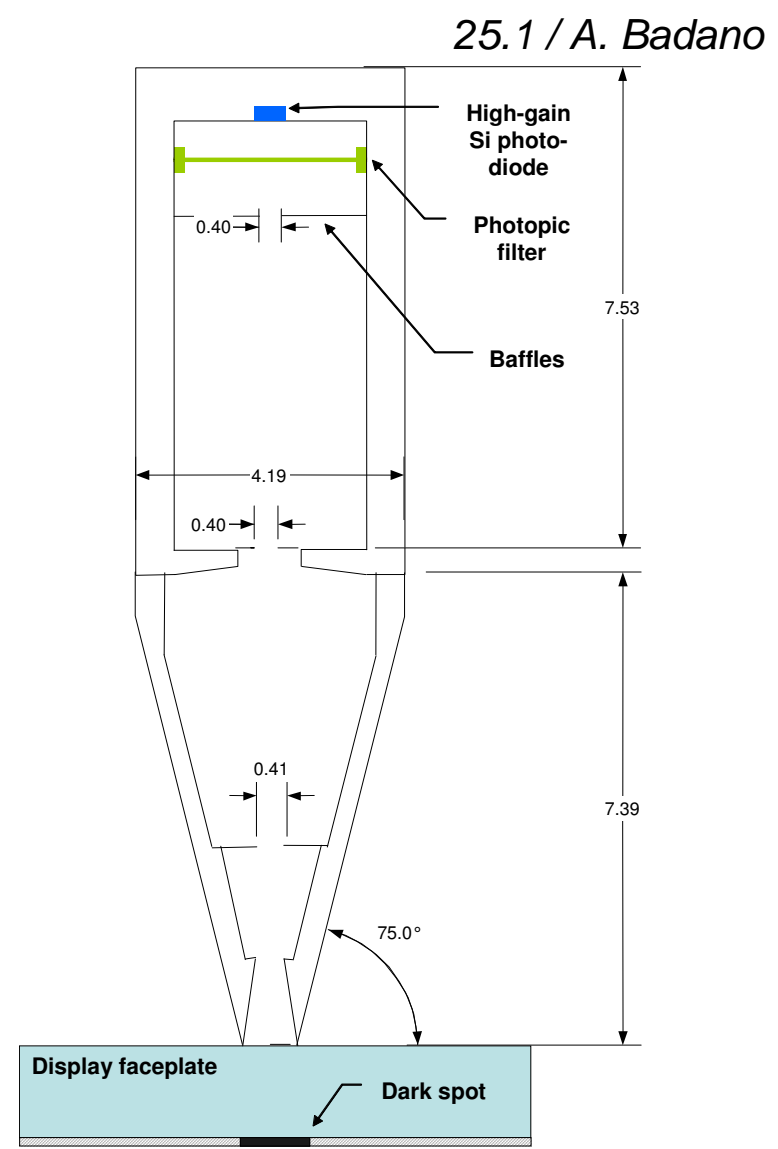

Fig. 2. Diagram showing the multiple baffles and conic entrance of the base design [8] used to fabricate the probes (dimensions in $\mathrm{cm}$ ). The probe is showed on top of a display with a thick faceplate.

through a 5-axis stage to obtain scan data automatically and reproducibly (only one axis was used to scan, the rest were used for alignment). Each probe was scanned along the $\mathrm{x}$ axis across the center of a plate in a direction perpendicular to a $100 \mu \mathrm{m}$ slit. The slit plate was made out of black vinyl plastic with a thickness of about $2 \mathrm{~mm}$. The actual slit was constructed from two razor blades tinted black. Placing such a slit in front of a uniform source provides us with a Lambertian output for all the angles of interest in this paper. The probes were positioned at distances $d_{z}$ of $1,2,4,8,16$ and $32 \mathrm{~mm}$ along the $\mathrm{z}$ axis away from the plate.

\section{RESULTS}

Fig. 3 shows the measured luminance scans for each of the four probes. Each of the graphs of the probe scans was normalized so that the maxima for each scan was centered at zero. The maximum luminance value for all the probes was present in the scan corresponding to a $d_{z}$ of $1 \mathrm{~mm}$. This maximum was used as the reference point for the 

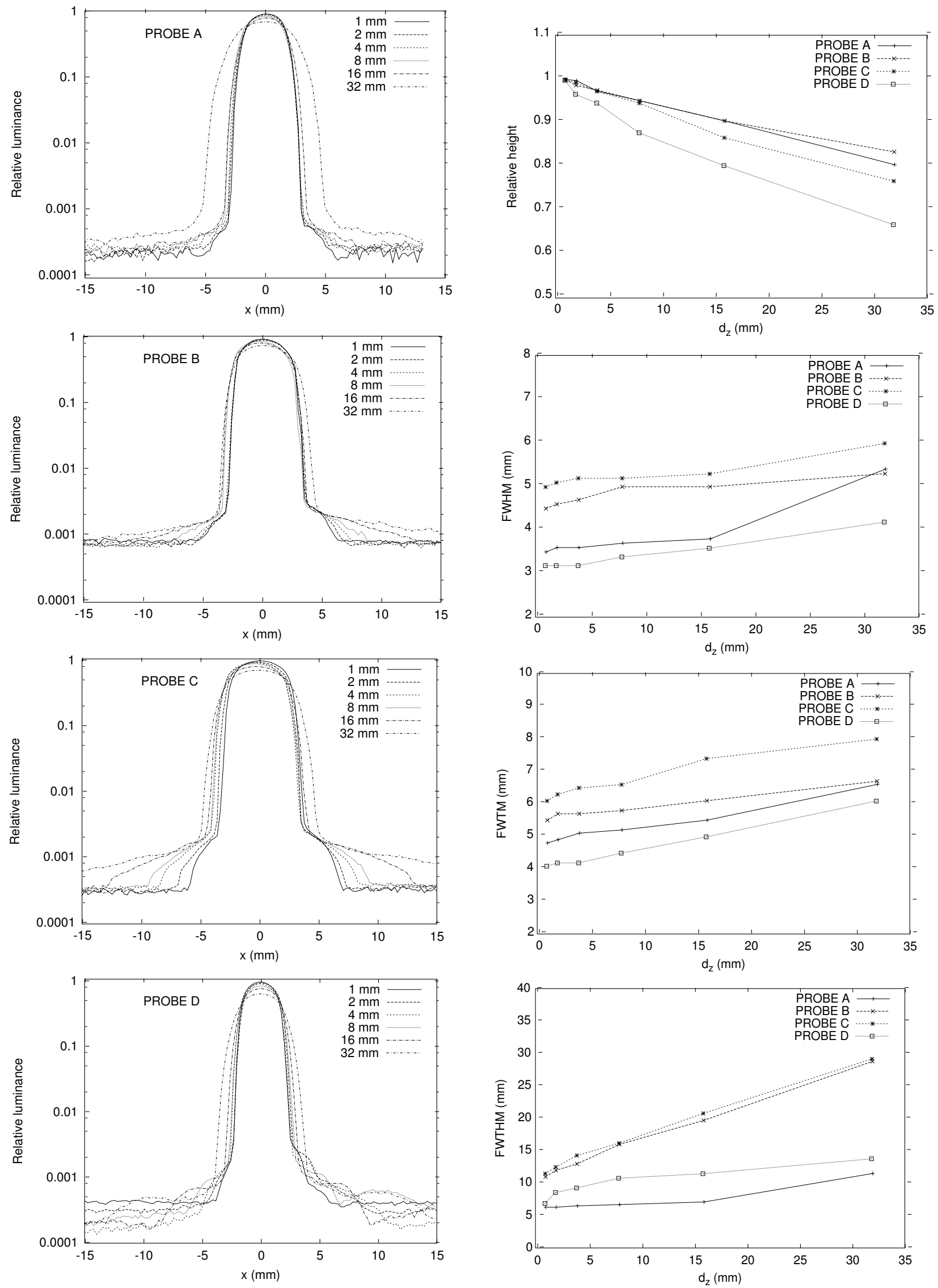

Fig. 3. Luminance scans of a $0.1 \mathrm{~mm}$ slit for six $d_{z}$ for the four Fig. 4. From top to bottom, comparison of scan heights, full-widthprobes studied in this work (A-D). at-half-maximum, full-width-at-tenth-maximum, and full-width-atthousandth-maximum. 
comparison with the other scans. The most notable differences observed in Fig. 3 is the presence of shoulder regions in both sides of the peak in the scans corresponding to probe $\mathrm{C}$ and $\mathrm{B}$ (to a lesser extent). For probes $\mathrm{A}$ and $\mathrm{D}$, the luminance falls rapidly until the measured signal reaches a constant level affected only by the noise of the measurement system. This level is about $3 \times 10^{-4}$ for both probes. The change in maximum luminance as a function of distance from the slit is approximately 0.15 for probe $\mathrm{B}, 0.20$ for $\mathrm{A}, 0.23$ for $\mathrm{C}$, and 0.35 for $\mathrm{D}$. We note that for probe $B$, the measurements at $32 \mathrm{~mm}$ have an intermediate region between the sharp slope and the plateau region.

The differences in minimum luminance are caused by differences in the probe's inner surface reflectance. Probe B was constructed out of black mylar which may have a slightly higher reflectivity than black anodized aluminum (probe $\mathrm{A}$ and $\mathrm{C}$ ) or black plastic (probe $\mathrm{D}$ ). In probe $\mathrm{C}$, one of the internal chambers has not been painted with flat black paint and remains highly reflective (nonanodized aluminum surface).

From the scans, we derived the height, full-widthat-half-maximum, full-width-at-tenth-maximum, and full-width-at-thousandth-maximum as a function of $d_{z}$. The results are presented in Fig. 4 in relative luminance units. All luminance values measured were at least one order of magnitude above the background level due to noise in the meter. Probe B maintains the height of the peak for longer distances between the probe and the plate, while probe $\mathrm{D}$ seems to suffer the most from this effect. The width of the peaks at different heights is presented also in Fig. 4. In this case, lower widths are representative of a better response to the slit luminance pattern. In the case of the full-width-at-half-maximum, probes $\mathrm{A}$ and $\mathrm{D}$ have the smaller widths. If we consider full-width-attenth-maximum, probes $\mathrm{D}$ results in the smaller widths. For the full-width-at-thousandth-maximum, probes $\mathrm{A}$ and $\mathrm{D}$ have slightly better performance than $\mathrm{B}$ and $\mathrm{C}$ by maintaining a small width up to $d_{z}=32 \mathrm{~mm}$.

\section{CONCLUSION}

This work proves that probes based on the same design can be constructed in different laboratories and achieve similar performance. Although we plan to investigate how small changes in the performance affect the measurements of display contrast with the different probes, we expect the effect to be small.

\section{ACKNOWLEDGEMENTS}

SP was supported in part by a Les Aspin Internship Program. The authors thank B. Fleharty (Tool Shop, FDA/CDRH) for providing for constructing several laboratory fixtures used during this work. We would like to also express our thanks to the Advanced Technology Program (ATP) of NIST and to E. I. Sobolewski (Program Manager, Electronics and Photonics Technology Office, ATP). The NIST contribution to this project was funded by the ATP.

\section{REFERENCES}

[1] D. Imaging, 2002, http://www.diagnosticimaging.com.

[2] E. Samei, A. Badano, D. Chakraborty, K. Compton, C. Cornelius, K. Corrigan, M. J. Flynn, B. Hemminger, N. Hangiandreou, J. Johnson, M. Moxley, W. Pavlicek, H. Roehrig, L. Rutz, J. Shepard, R. Uzenoff, J. Wang, and C. Willis, Assessment of Display Performance for Medical Imaging Systems. Draft Report of the American Association of Physicists in Medicine Task Group 18, Version 9.1. American Association of Physicists in Medicine, 2003.

[3] A. Badano, M. J. Flynn, E. Muka, K. Compton, and T. Monsees, "Veiling glare point-spread function of medical imaging monitors," Proceedings of the 1999 Medical Imaging - Image Display, vol. 3658, pp. 458-467, 1999.

[4] —, "The veiling glare point-spread function of medical imaging monitors," in Medical Imaging 1999: Image Display, 1999, pp. 458-67.

[5] A. Badano, M. J. Flynn, and J. Kanicki, "Accurate smallspot luminance measurements," Displays, vol. 23, pp. 177-182, 2002.

[6] S. Martin, A. Badano, and J. Kanicki, "High-resolution medical imaging AM-LCD: Contrast performance evaluation," in Proceedings Of International Display Research Conference, vol. 4681. SID, 2002.

[7] — "Characterization of a high quality monochrome AMLCD monitor for digital radiology," in Proceedings Of Medical Imaging Conference: Image Display, vol. 4681. SPIE, 2002, pp. 293-304.

[8] A. Badano and M. J. Flynn, "A method for measuring veiling glare in high performance display devices," Applied Optics, vol. 39, no. 13, pp. 2059-2066, May 2000.

[9] E. F. Kelley and A. Badano, "Characterization of luminance probe for accurate contrast measurements in medical displays," To be published as a NISTIR (National Institute of Standards and Technology Interagency Report), 2002.

[10] Certain commercial equipment, instruments, materials, systems, and trade names are identified in this paper in order to specify or identify technologies adequately. Such identification is not intended to imply recommendation or endorsement by the National Institute of Standards and Technology nor the Food and Drug Administration, nor is it intended to imply that the systems or products identified are necessarily the best available for the purpose.

[11] E. F. Kelley, "Polystyrene box uniform light sources," in CORM'99, 1999, http://www.fpdl.nist.gov/sources.html. 